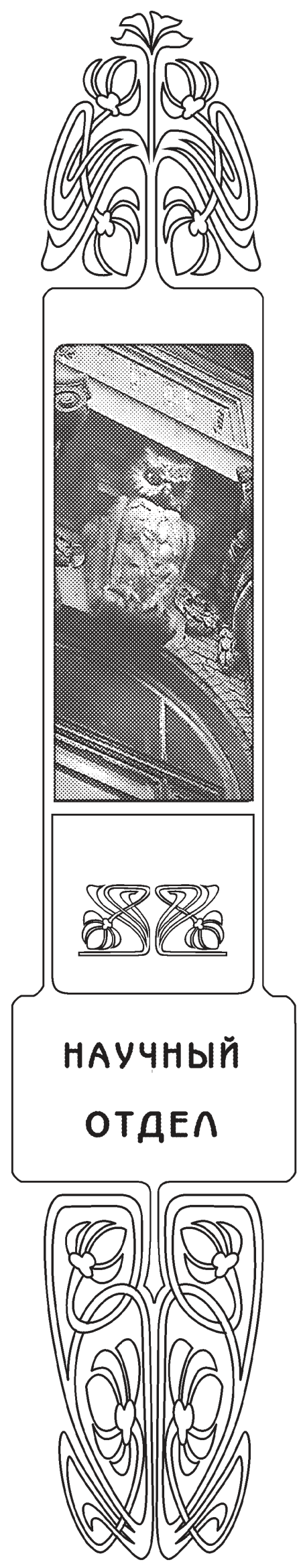

\title{
MEХАНИКА
}

\section{Representation of Waves of Displacements and Micro-rotations by Systems of the Screw Vector Fields}

\section{Yu. N. Radayev}

Yuri N. Radayev, https://orcid.org/0000-0002-0866-2151, Institute for Problems in Mechanics of RAS (IPMech RAS), 101 Vernadskogo Ave., Moscow 119526, Russia, y.radayev@gmail.com

The present study concerns the coupled vector differential equations of the linear theory of micropolar elasticity formulated in terms of displacements and micro-rotations in the case of a harmonic dependence of the physical fields on time. The system is known from many previous discussions on the micropolar elasticity. A new analysis aimed at uncoupling the coupled vector differential equation of the linear theory of micropolar elasticity is carried out. A notion of proportionality of the vortex parts of the displacements and microrotations to a single vector, which satisfies the screw equation, is employed. Finally the problem of finding the vortex parts of the displacements and micro-rotations fields is reduced to solution of four uncoupled screw differential equations. Corresponding representation formulae are given. Obtained results can be applied to problems of the linear micropolar elasticity concerning harmonic waves propagation along cylindrical waveguides.

Keywords: micropolar elasticity, displacement vector, micro-rotation vector, coupled, vector potential, vortex part, screw equation, screw field, Helmholtz equation, waveguide.

Received: 11.05.2020 / Accepted: 28.06.2020 /

Published: 30.11 .2020

This is an open access article distributed under the terms of Creative Commons Attribution License (CC-BY 4.0)

DOI: https://doi.org/10.18500/1816-9791-2020-20-4-468-477

\section{INTRODUCTORY REMARKS}

The micropolar theory of elasticity, for the first time proposed in the classical work [1], is nowdays considered as a natural generalisation of the theory of elasticity, well known as both engineering and physical science $[2,3]$. The micropolar theory of elasticity offers its own recipe for overcoming 
the difficulties encountered by the theory of elasticity in attempts, for example, to explain the mechanical behaviour of granular media or patterns of high-frequency acoustic waves propagation through polycrystalline structures. The equations of the linear micropolar elasticity theory are well established and discussed (see [4,5]). Their derivation, based on the principle of virtual displacements, is considered in the paper [6].

The aim of the present work is to study the coupled system of vector differential equations of the linear micropolar theory of an isotropic elastic body in the case of the harmonic dependence of displacement and micro-rotation fields on time. The system is coupled with respect to displacement and micro-rotation fields. Their study and transformation by the aid of dynamic potentials (vortex-free and vortex) lead to various interesting systems of vector differential equations (both coupled and uncoupled). From both the theoretical and applied point of views, the most interesting ones are those that provide a transition from coupled equations to uncoupled ones. Related problems and problem formulations also arise in applied problems of the coupled thermoelasticity [7], especially in the case of propagation of harmonic waves in hyperbolic thermoelastic media (see [8] for further details).

In this paper an alternative scheme for splitting the main coupled system of vector differential equations of the harmonic micropolar elasticity into uncoupled equations is developed. The latter will take the form of screw equations. The minimum background information concerning such equations is given below (see also, for example, [9]).

Vector field $\Upsilon$ in three-dimensional space is called a screw field if it satisfies the following equation:

$$
\Upsilon \times(\nabla \times \Upsilon)=0,
$$

i.e. the vortex of the vector field $\Upsilon$ is to be colinear to the direction of the field itself

$$
\nabla \times \Upsilon=A \Upsilon
$$

wherein the scalar multiplier $A$ is discriminated by the term abnormality of the field.

If the multiplier $A$ in (1) has a constant value then:

1) all multiples of the vortex vector field $\Upsilon$ are defined according to

$$
\boldsymbol{\nabla} \times \Upsilon, \quad \boldsymbol{\nabla} \times(\boldsymbol{\nabla} \times \Upsilon), \quad \boldsymbol{\nabla} \times(\boldsymbol{\nabla} \times(\boldsymbol{\nabla} \times \Upsilon)), \ldots
$$

are also screw fields with the same abnormality $A$;

2) screw vector field $\Upsilon$ satisfies the vector Helmholtz differential equation

$$
\Delta \Upsilon+A^{2} \Upsilon=0
$$

3) screw vector field $\Upsilon$ with the constant abnormality $A$ can be represented in the following form

$$
\Upsilon=A(\nabla h) \times \mathbf{d}+A^{2} h \mathbf{d}+(\mathbf{d} \cdot \boldsymbol{\nabla}) \boldsymbol{\nabla} h
$$

where $\mathbf{d}-$ a constant unit director in three-dimensional space, $h-$ a scalar field which satisfies the scalar Helmholtz differential equation

$$
\Delta h+A^{2} h=0 .
$$




\section{DIFFERENTIAL EQUATIONS OF THE LINEAR THEORY OF MICROPOLAR ELASTICITY IN TERMS OF DISPLACEMENTS AND MICRO-ROTATIONS}

The system of coupled vector differential equations of linear micropolar elasticity theory has the following form [5]:

$$
\left\{\begin{aligned}
G\left[\left(1+c_{1}\right) \boldsymbol{\nabla} \cdot \boldsymbol{\nabla u}+\left(1-c_{1}+2 \nu(1-2 \nu)^{-1}\right) \boldsymbol{\nabla} \boldsymbol{\nabla} \cdot \mathbf{u}+2 c_{1} \boldsymbol{\nabla} \times \boldsymbol{\phi}\right] & =\rho \partial . \partial . \mathbf{u}, \\
G L^{2}\left[\left(1+c_{2}\right) \boldsymbol{\nabla} \cdot \boldsymbol{\nabla} \boldsymbol{\phi}+\left(1-c_{2}+2 c_{3}\right) \boldsymbol{\nabla} \boldsymbol{\nabla} \cdot \boldsymbol{\phi}\right]-2 G c_{1}(2 \boldsymbol{\phi}-\boldsymbol{\nabla} \times \mathbf{u}) & =\Im \partial . \partial . \boldsymbol{\phi}
\end{aligned}\right.
$$

wherein $\rho$ - mass density; $\mathfrak{I}$ - microinertia coefficient; $\mathbf{u}-$ displacement vector; $\phi-$ micro-rotation vector; $G$ - shear modulus of elasticity; $\nu$ - Poisson ratio; $L-$ characteristic length of the micropolar elasticity theory; $c_{1}, c_{2}, c_{3}$ - dimensionless constitutive constants of the micropolar elasticity; $\boldsymbol{\nabla}$ - three-dimensional nabla-operator; $\partial$. - partial time differentiation when the spatial variables are fixed.

The above system of vector partial differential equations (2) seems to be the most acceptable from a physical point of view. However, it is not widely used in modern discussions on the micropolar elasticity, since a different set of constitutive constants is usually considered as optimal. For this reason, we introduce new constitutive constants $\alpha, \beta, \gamma, \lambda, \mu, \varepsilon$ according to

$$
\begin{gathered}
G=\mu, \quad \frac{2 \nu}{1-2 \nu}=\frac{\lambda}{\mu}, \quad G L^{2}=\gamma, \\
c_{1}=\frac{\alpha}{\mu}, \quad c_{2}=\frac{\varepsilon}{\gamma}, \quad c_{3}=\frac{\beta}{2 \gamma} .
\end{gathered}
$$

Note that they are systematically used in the monographs by W. Nowacki $[4,5]$ and his many other publications devoted to the linear micropolar theory of elasticity of an isotropic body. As a result, the given system of coupled equations of linear micropolar elasticity theory (2) is represented in the following form:

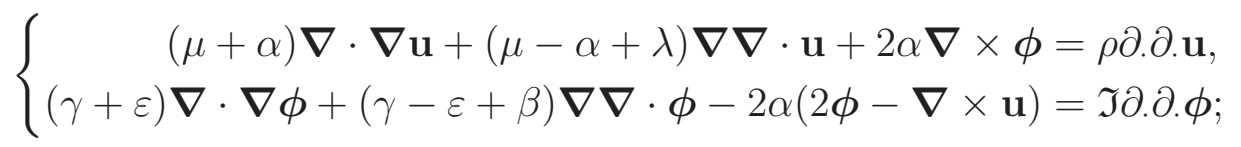

or

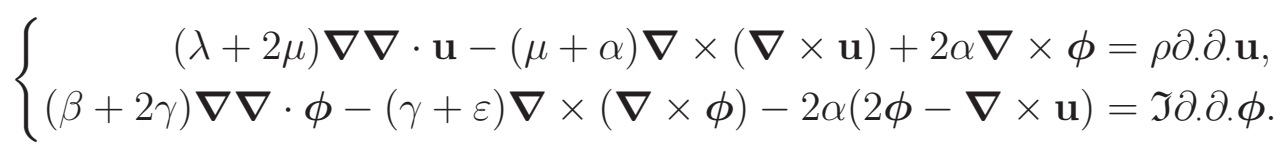

The coupled vector differential equations (4) have been the subject of numerous discussions. Among them the following early papers [10-13] and the monograph [14] should be mentioned.

In the following discussion the vector differential equations (4) will be considered in domains of three-dimensional space that have the property of surface simply connected, i.e. any closed surface situated entirely within the domain can be drawn to a point without going beyond the boundary of the domain. This requirement is absolutely necessary in order for any vortex-free vector field to be a potential, and any vector field with zero divergence to be a vortex, i.e., to have a vector potential.

The time dependence is assumed to be harmonic, i.e. physical fields are represented as products of complex amplitudes (for which we keep the same notation as for the fields themselves) by the complex harmonic exponent $e^{i \omega t}$, where $\omega$ is the cyclic frequency. 


\section{DISPLACEMENT AND MICRO-ROTATION POTENTIALS. COUPLED EQUATIONS FOR THE POTENTIALS} vectors

We employ the Helmholtz decomposition for the displacement and micro-rotation

$$
\begin{aligned}
& \mathbf{u}=\nabla \Phi+\nabla \times \mathbf{\Psi}, \\
& \phi=\nabla \Sigma+\nabla \times \mathbf{H},
\end{aligned}
$$

which represent these vector fields by using the pairs: scalar potentials $\Phi, \Sigma$, and vector potentials $\boldsymbol{\Psi}, \mathbf{H}$. Such a decomposition is discussed, for instance, in [7].

One may complement (or may not complement) various calibration conditions to (5). In particular, condition stating zero divergence of vector potentials is usually considered as standard

$$
\boldsymbol{\nabla} \cdot \boldsymbol{\Psi}=0, \quad \boldsymbol{\nabla} \cdot \mathbf{H}=0 .
$$

Substitution of (5) decompositions into the system of differential equations (4) allows us to get equations for the scalar and vector potentials.

The differential equations for scalar potentials $\Phi, \Sigma$ are not coupled and therefore can be considered as the two independent equations

$$
\begin{aligned}
& \Delta \Phi-\frac{1}{c_{\|}^{2}}(\partial .)^{2} \Phi=0, \\
& \Delta \Sigma-\frac{1}{{ }_{\mu} c_{\|}^{2}}(\partial .)^{2} \Sigma-\frac{\Omega^{2}}{{ }_{\mu}^{2}} \Sigma=0 .
\end{aligned}
$$

Here the constants $c_{\|}^{2},{ }_{\mu} c_{\|}^{2}$ and $\Omega^{2}$ are expressed in terms of the constitutive constants according to

$$
c_{\|}^{2}=\frac{\lambda+2 \mu}{\rho}, \quad{ }_{\mu} c_{\|}^{2}=\frac{\beta+2 \gamma}{\mathfrak{I}}, \quad \Omega^{2}=\frac{4 \alpha}{\mathfrak{I}} .
$$

For vector potentials $\boldsymbol{\Phi}, \mathbf{H}$ the two coupled vector differential equations are obtained

$$
\left\{\begin{array}{r}
\mathscr{A}_{\perp} \boldsymbol{\Psi}+2 d_{\perp}^{2} \boldsymbol{\nabla} \times \mathbf{H}=\mathbf{0}, \\
\mathscr{B}_{\perp} \mathbf{H}+\frac{\Omega^{2}}{2{ }_{\mu}{ }_{\mu}^{2} c_{\perp}^{2}} \boldsymbol{\nabla} \times \boldsymbol{\Psi}=\mathbf{0},
\end{array}\right.
$$

where the following constants have been introduced

$$
d_{\perp}^{2}=\frac{{ }^{\prime} c_{\perp}^{2}}{{ }^{\prime} c_{\perp}^{2}}, \quad{ }^{\prime} c_{\perp}^{2}=\frac{\alpha}{\rho}, \quad "{ }^{2} c_{\perp}^{2}=\frac{\mu+\alpha}{\rho}, \quad{ }_{\mu}^{\prime \prime} c_{\perp}^{2}=\frac{\gamma+\epsilon}{\mathfrak{I}} ;
$$

and the two second order differential operators

$$
\mathscr{A}_{\perp}=\Delta-\frac{1}{{ }^{\prime} c_{\perp}^{2}}(\partial .)^{2}, \quad \mathscr{B}_{\perp}=\Delta-\frac{1}{{ }_{\mu} c_{\perp}^{2}}(\partial .)^{2}-\frac{\Omega^{2}}{{ }_{\mu} c_{\perp}^{2}} .
$$

The system (7) keeps its form regardless of the use of a particular calibration condition. This means that the calibration conditions (6) may be abandoned altogether.

A study of the coupled equations for vector potentials of displacements and microrotations causes considerable difficulties and therefore we will focus on these equations. 
Let us start with the fact that the differential operators $\mathscr{A}_{\perp}$ and $\mathscr{B}_{\perp}$ in the case of harmonic dependence on time are

$$
\mathscr{A}_{\perp}=\Delta+\frac{\omega^{2}}{{ }^{\prime} c_{\perp}^{2}}, \quad \mathscr{B}_{\perp}=\Delta+\frac{\omega^{2}}{{ }_{\mu} c_{\perp}^{2}}-\frac{\Omega^{2}}{{ }_{\mu} c_{\perp}^{2}} .
$$

In this study it is convenient to introduce the following two constants

$$
\alpha_{\perp}^{2}=\frac{\omega^{2}}{{ }^{\prime \prime} c_{\perp}^{2}}, \quad \beta_{\perp}^{2}=\operatorname{Abs} \frac{\omega^{2}-\Omega^{2}}{{ }_{\mu}^{11} c_{\perp}^{2}}
$$

and then reduce the differential operators $\mathscr{A}_{\perp}$ and $\mathscr{B}_{\perp}$ to

$$
\mathscr{A}_{\perp}=\Delta+\alpha_{\perp}^{2}, \quad \mathscr{B}_{\perp}=\Delta \pm \beta_{\perp}^{2},
$$

where the choice of a sign in the expression for $\mathscr{B}_{\perp}$ depends on the magnitude of the cyclic frequency

$$
\omega^{2}-\Omega^{2} \gtrless 0 .
$$

As a result, in the harmonic case, the coupled system of equations for potentials takes the form

$$
\left\{\begin{array}{c}
\left(\Delta+\alpha_{\perp}^{2}\right) \Psi+2 d_{\perp}^{2} \nabla \times \mathbf{H}=\mathbf{0}, \\
\left(\Delta \pm \beta_{\perp}^{2}\right) \mathbf{H}+\frac{\Omega^{2}}{2{ }_{\mu}^{\prime \prime} c_{\perp}^{2}} \nabla \times \mathbf{\Psi}=\mathbf{0} .
\end{array}\right.
$$

The following discussion will be restricted to the study of high-frequency harmonic waves when the cyclic frequency $\omega$ is higher than the threshold value determined by the constant $\Omega$ (see (12)). Then the latter system of equations is reduced to

$$
\left\{\begin{array}{c}
\left(\Delta+\alpha_{\perp}^{2}\right) \mathbf{\Psi}+2 d_{\perp}^{2} \nabla \times \mathbf{H}=\mathbf{0}, \\
\left(\Delta+\beta_{\perp}^{2}\right) \mathbf{H}+\frac{\Omega^{2}}{2{ }_{\mu}^{\prime \prime} c_{\perp}^{2}} \nabla \times \mathbf{\Psi}=\mathbf{0} .
\end{array}\right.
$$

By introducing the notation

$$
g_{\perp}^{2}=\frac{\Omega^{2}}{{ }_{\mu}^{11} c_{\perp}^{2}} d_{\perp}^{2},
$$

we finally come to the following system of equations:

$$
\left\{\begin{array}{l}
\left(\Delta+\alpha_{\perp}^{2}\right) \mathbf{\Psi}+2 d_{\perp}^{2} \nabla \times \mathbf{H}=\mathbf{0}, \\
\left(\Delta+\beta_{\perp}^{2}\right) \mathbf{H}+\frac{g_{\perp}^{2}}{2 d_{\perp}^{2}} \nabla \times \mathbf{\Psi}=\mathbf{0} .
\end{array}\right.
$$

A careful analysis of the reasonings shows that the coupled system of equations for potentials (15) is also obtained in a slightly different form (and again without taking account of the calibration of potentials):

$$
\left\{\begin{array}{l}
-\boldsymbol{\nabla} \times(\boldsymbol{\nabla} \times \mathbf{\Psi})+\alpha_{\perp}^{2} \boldsymbol{\Psi}+2 d_{\perp}^{2} \boldsymbol{\nabla} \times \mathbf{H}=\mathbf{0}, \\
-\boldsymbol{\nabla} \times(\boldsymbol{\nabla} \times \mathbf{H})+\beta_{\perp}^{2} \mathbf{H}+\frac{g_{\perp}^{2}}{2 d_{\perp}^{2}} \boldsymbol{\nabla} \times \boldsymbol{\Psi}=\mathbf{0} .
\end{array}\right.
$$




\section{REPRESENTATION OF VORTEX PARTS OF DISPLACEMENTS AND MICRO-ROTATIONS BY SCREW VECTOR FIELDS}

The representation of vortex parts of displacements and micro-rotations by using screw vector fields solves the principal problem of our study: the transition from the coupled system of differential equations (16) to uncoupled equations, which should eventually allow us to find out analytical approaches to investigation of applied problems in the mechanics of micropolar continua.

Achieving this goal begins with considering the vortex parts of displacements and micro-rotations as a vortex vector field $\Upsilon$ with different scale factors:

$$
\left\{\begin{array}{l}
\boldsymbol{\nabla} \times \boldsymbol{\Psi}=a \Upsilon \\
\boldsymbol{\nabla} \times \mathbf{H}=b \Upsilon
\end{array}\right.
$$

This will meet the natural calibration condition discussed earlier:

$$
\nabla \cdot \Upsilon=0
$$

Upon substituting (17) into the system of vector differential equations (16) the following system of equations with respect to the field $\Upsilon$ is obtained:

$$
\left\{\begin{array}{l}
-a \boldsymbol{\nabla} \times(\boldsymbol{\nabla} \times \boldsymbol{\Upsilon})+\alpha_{\perp}^{2} a \boldsymbol{\Upsilon}+2 d_{\perp}^{2} b \boldsymbol{\nabla} \times \boldsymbol{\Upsilon}=\mathbf{0} \\
-b \boldsymbol{\nabla} \times(\boldsymbol{\nabla} \times \boldsymbol{\Upsilon})+\beta_{\perp}^{2} b \boldsymbol{\Upsilon}+\frac{g_{\perp}^{2}}{2 d_{\perp}^{2}} a \boldsymbol{\nabla} \times \boldsymbol{\Upsilon}=\mathbf{0} .
\end{array}\right.
$$

In the left side of the first equations of the above system we add and subtract the same summand ( $c$ is a constant)

$$
c \nabla \times \Upsilon
$$

we do the same with the second equation and the summand ( $d$ is a constant)

$$
d \nabla \times \Upsilon .
$$

After a series of transformations, we make sure that if we put

$$
\frac{a}{c}=\frac{c+2 d_{\perp}^{2} b}{a \alpha_{\perp}^{2}}, \quad \frac{b}{d}=\frac{d+\left(2 d_{\perp}^{2}\right)^{-1} g_{\perp}^{2} a}{b \beta_{\perp}^{2}},
$$

then the coupled equations for potentials are satisfied when

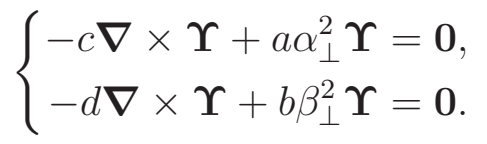

One can obtain single independent equation for determining the vortex vector field $\Upsilon$ if

$$
\frac{c}{d}=\frac{\alpha_{\perp}^{2}}{\beta_{\perp}^{2}} \frac{a}{b}
$$

After that it is sufficient the following screw equation to be satisfied

$$
-\nabla \times \Upsilon+p \alpha_{\perp}^{2} \Upsilon=\mathbf{0}
$$


where $p$ is defined as the ratio

$$
p=\frac{a}{c} .
$$

We proceed to finding constants, involved in our reasoning. There are four constants in total: $a, b, c, d$. Three independent ratios can be formed from them:

$$
p=\frac{a}{c}, \quad q=\frac{b}{c}, \quad s=\frac{d}{c}
$$

For these ratios exactly three independent equations are obtained from the previous arguments

$$
\left\{\begin{array}{l}
p^{2} \alpha_{\perp}^{2}=1+2 q d_{\perp}^{2}, \\
q^{2} \beta_{\perp}^{2}=s^{2}+p s \frac{g_{\perp}^{2}}{2 d_{\perp}^{2}}, \\
p s \frac{\alpha_{\perp}^{2}}{\beta_{\perp}^{2}}=q .
\end{array}\right.
$$

From the above system of equations one can determine the constant $q$ by the quadratic equation

$$
2 d_{\perp}^{2} \alpha_{\perp}^{2} q^{2}+\left(\alpha_{\perp}^{2}-\beta_{\perp}^{2}-g_{\perp}^{2}\right) q-\frac{g_{\perp}^{2}}{2 d_{\perp}^{2}}=0
$$

where the two different real values for $q$ can be found:

$$
4 d_{\perp}^{2} \alpha_{\perp}^{2} q=\beta_{\perp}^{2}+g_{\perp}^{2}-\alpha_{\perp}^{2} \pm \sqrt{\left(\beta_{\perp}^{2}+g_{\perp}^{2}-\alpha_{\perp}^{2}\right)^{2}+4 g_{\perp}^{2} \alpha_{\perp}^{2}}
$$

For the constant $p$ there are two different real values as well

$$
2 \alpha_{\perp}^{4} p^{2}=\beta_{\perp}^{2}+g_{\perp}^{2}+\alpha_{\perp}^{2} \pm \sqrt{\left(\beta_{\perp}^{2}+g_{\perp}^{2}+\alpha_{\perp}^{2}\right)^{2}-4 \alpha_{\perp}^{2} \beta_{\perp}^{2}}
$$

Starting from this point we introduce the two real values $p_{1}, p_{2}$, which correspond to the positive and negative signs in the formula given above, and the two values (wavenumbers) $K_{1}, K_{2}$ according to

$$
\alpha_{\perp}^{4} p_{1,2}^{2}=K_{2,1}^{2}, \quad \alpha_{\perp}^{2} p_{1}=\mp K_{2}, \quad \alpha_{\perp}^{2} p_{2}=\mp K_{1} .
$$

Note that the wavenumbers $K_{1}, K_{2}$ are ordered on the ground of their definition as follows

$$
K_{2}>K_{1}>0 .
$$

Thus there are the four different real values for the parameter $p$ :

$$
\mp K_{1}, \quad \mp K_{2} .
$$

Consequently, the vector field $\Upsilon$ must satisfy one of the four screw equations

$$
-\nabla \times \Upsilon \mp K_{2,1} \Upsilon=\mathbf{0},
$$

where the signs $\mp$ and indices 1,2 are in no way co-ordinated with each other. 
It means that there are exactly four independent fields ${ }^{1}$

$$
\Upsilon_{2}, \quad \Upsilon_{2}, \quad \Upsilon_{-}, \quad \Upsilon_{1},
$$

which must be integrals of the uncoupled vector equations (19) and whose linear combinations are to be the vortex parts of the displacement and micro-rotation vectors in accordance with (17). It should be noted that only the ratio $b / a$ is uniquely defined and since

$$
\frac{b}{a}=\frac{q}{p},
$$

the ratio $b / a$ has four different real values, which are easily determined from the above formulae.

By denoting the four mentioned values of the ratio $b / a$ as

$$
\frac{b}{a}=\mp g_{2,1},
$$

finally we come to the following representation formula for the vortex parts of the displacement and micro-rotation vectors:

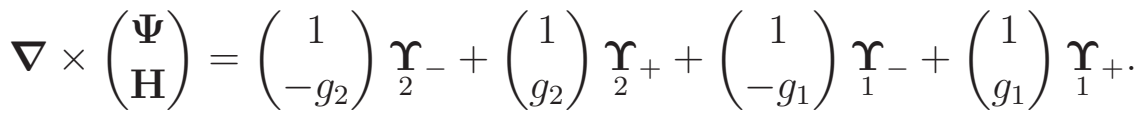

The completeness of the representations of harmonic fields of displacements and micro-rotations by using four screw vector fields considered in this paper can be established by the known (and comparatively simple) methods.

The results obtained in this paper are aimed at their use in applied problems on propagation of harmonic (monochromatic) waves of displacements and micro-rotations along cylindrical waveguides and in this sense are to be treated as a further development of the methods and results given in the book [8].

\section{CONCLUSIONS}

1. The system of coupled vector differential equations of the linear theory of micropolar elasticity formulated in terms of displacements and micro-rotations in the case of the harmonic dependence of physical fields on time (monochromatic waves of displacements and micro-rotations) has been studied.

2. Uncoupled equations for scalar and vector potentials, which determine displacement and micro-rotation fields in accordance with the Lame representation, have been obtained. It has been demonstrated that vector solutions of the uncoupled equations provide solutions of the coupled vector differential equations of the linear theory of micropolar elasticity.

3. An alternative scheme of uncoupling vector differential equations of the micropolar elasticity theory for potentials has been developed. It is based on the proportionality of the vortex parts of displacements and micro-rotations to a single vortex screw field.

4. The problem of finding the vortex parts of displacements and micro-rotations has been reduced to the solution of four uncoupled screw vector partial differential equations of the first order.

\footnotetext{
${ }^{1}$ The following notations for the four different variants of the vector field $\Upsilon$ is consistent to the four equations, written in the compact form (19).
} 
5. A representation of displacement and micro-rotation vectors has been obtained by using four screw vectors thus providing the coupled vector differential equations of the linear theory of micropolar elasticity to be satisfied.

6. Completeness of the considered in this paper representations of the harmonic fields of displacements and micro-rotations has been discussed involving the known and rather simple methods by employing four screw vector fields.

7. The results of the present paper can be used in applied problems related to the propagation of harmonic (monochromatic) waves of displacement and micro-rotation along long cylindrical waveguides.

Acknowledgements: This work was in part financially supported by the Ministry of Science and Higher Education of the Russian Federation (State Registration Number AAAA-A20-120011690132-4) and by the Russian Foundation for Basic Research (project No. 18-01-00844).

\section{References}

1. Cosserat E., Cosserat F. Theorie des corps deformables. Paris, Herman et Fils, 1909. $226 \mathrm{p}$.

2. Southwell R. V. An Introduction to the Theory of Elasticity for Engineers and Physicists. Oxford Engineering Science Series. Oxford, Clarendon Press; London, Oxford University Press, 1936. 510 p.

3. Lurie A. I. Theory of Elasticity. Berlin, Heidelberg, Springer, 2005. 1050 p.

4. Nowacki W. Theory of Asymmetric Elasticity. Oxford, New York, Toronto, Sydney, Paris, Frankfurt, Pergamon Press, 1986. 383 p.

5. Nowacki W. Theory of Elasticity. Moscow, Mir, 1975. 872 p. (in Russian).

6. Radayev Yu. N. The Lagrange multipliers method in covariant formulations of micropolar continuum mechanics theories. Vestn. Samar. Gos. Tekhn. Univ., Ser. Fiz.-Mat. Nauki [J. Samara State Tech. Univ., Ser. Phys. Math. Sci.], 2018, vol. 22, no. 3, pp. 504-517 (in Russian). DOI: https://doi.org/10.14498/vsgtu1635

7. Achenbach J. D. Wave Propagation in Elastic Solids. North-Holland Series in Applied Mathematics and Mechanics. Vol. 16. Amsterdam, London, North-Holland; New York, American Elsevier, 1973. 425 p.

8. Kovalev V. A., Radayev Yu. N. Volnovye zadachi teorii polia [Wave Problems of the Field Theory]. Saratov, Izd-vo Saratovskogo universiteta, 2010. 328 p. (in Russian).

9. Truesdell C., Toupin R. The Classical Field Theories. In: S. Flugge (ed.). Encyclopedia of Physics. Vol. III/1. Principles of Classical Mechanics and Field Theory. Berlin, Gottingen, Heidelberg, Springer, 1960. P. 226-902.

10. Gunter W. Zur Statik und Kinematik des Cosseratschen Kontinuums. Abhandlungen der Braunschweigischen Wissenschaftlichen Gesellschaft, 1958, Bd. 10, s. 195-213 (in Germany).

11. Kessel S. Lineare Elastizitatstheorie des anisotropen Cosserat-Kontinuums. Abhandlungen der Braunschweigischen Wissenschaftlichen Gesellschaft, 1964, Bd. 16, s. 1-22 (in Germany).

12. Pal'mov V. A. Fundamental equations of the theory of asymmetric elasticity. J. Appl. Math. Mech., 1964, vol. 28, iss. 3, pp. 496-505. DOI: https://doi.org/10.1016/00218928(64)90092-9

13. Neuber H. Über Probleme der Spannungskonzentration im Cosserat-Körper. Acta Mechanica, 1966, vol. 2, iss. 1, pp. 48-69 (in Germany). DOI: https://doi.org/10.1007/ BF01176729

14. Dyszlewicz J. Micropolar Theory of Elasticity. Lecture Notes in Applied and Computational Mechanics. Berlin, Heidelberg, Springer, 2004. 345 p. 


\title{
Cite this article as:
}

Radayev Yu. N. Representation of Waves of Displacements and Micro-rotations by Systems of the Screw Vector Fields. Izv. Saratov Univ. (N. S.), Ser. Math. Mech. Inform., 2020, vol. 20, iss. 4, pp. 468-477. DOI: https://doi.org/10.18500/1816-9791-2020-20-4-468-477

\section{УДК 539.3}

\section{Представление волн перемещений и микровращений системами винтовых векторов}

\begin{abstract}
Ю. Н. Радаев
Радаев Юрий Николаевич, доктор фризико-математических наук, профрессор, ведущий научный сотрудник, Институт проблем механики имени А. Ю. Ишлинского РАН (ИПМех РАН), Россия, 119526, г. Москва, просп. Вернадского, д. 101, корп. 1, y.radayev@gmail.com

Рассматриваются диффреренциальные уравнения линейной микрополярной теории упругости в случае гармонической зависимости поля перемещений и микровращений от времени, из которой выводятся связанные уравнения для потенциалов. Предложена новая схема расщепления связанных векторных диффреренциальных уравнений микрополярной теории упругости для потенциалов на несвязанные диффреренциальные уравнения первого порядка. Получено представление векторов перемещений и микровращений с помощью системы четырех винтовых векторов, обеспечивающее выполнимость связанных векторных дифрференциальных уравнений, после чего проблема определения вихревых составляющих перемещений и микровращений сводится $\mathrm{K}$ решению четырех не связанных между собой векторных винтовых диффреренциальных уравнений первого порядка с частными производными. Указанное представление пригодно для использования в прикладных задачах механики, связанных с распространением пространственных гармонических волн перемещений и микровращений вдоль длинных волноводов.
\end{abstract}

Ключевые слова: микрополярная теория упругости, вектор перемещения, вектор микровращения, связанный, векторный потенциал, вихревая часть, винтовое уравнение, винтовое поле, уравнение Гельмгольца, волновод.

Поступила в редакцию: 11.05.2020 / Принята: 28.06.2020 / Опубликована: 30.11.2020

Статья опубликована на условиях лицензии Creative Commons Attribution License (CC-BY 4.0)

Благодарности. Работа выполнена при частичной финансовой поддержке Министерства науки и высшего образования Российской Федерации (государственный регистрационный номер АААА-А20-120011690132-4) и РФФИ (проект № 18-01-00844).

\section{Образец для цитирования:}

Radayev Yu. N. Representation of Waves of Displacements and Micro-rotations by Systems of the Screw Vector Fields [Радаев Ю. Н. Представление волн перемещений и микровращений системами винтовых векторов] // Изв. Сарат. ун-та. Нов. сер. Сер. Математика. Механика. Информатика. 2020. Т. 20, вып. 4. C. 468-477. DOI: https://doi.org/10.18500/1816-97912020-20-4-468-477 